\title{
Narrow-Band Imaging System for the Multi-application Solar Telescope at Udaipur Solar Observatory: Characterization of Lithium Niobate Etalons
}

\author{
A. Raja Bayanna - Shibu K. Mathew • \\ P. Venkatakrishnan • N. Srivastava
}

(C) Springer $\bullet \bullet \bullet \bullet$

\begin{abstract}
Multi-application Solar Telescope is a $50 \mathrm{~cm}$ off-axis Gregorian telescope that has been installed at the lake site of Udaipur Solar Observatory. For quasi-simultaneous photospheric and chromospheric observations, a narrow-band imager has been developed as one of the back-end instruments for this telescope. Narrow-band imaging is achieved using two lithium niobate Fabry-Perot etalons working in tandem as a filter. This filter can be tuned to different wavelengths by changing either voltage, tilt or temperature of the etalons. To characterize the etalons, a Littrow spectrograph was set up, in conjunction with a $15 \mathrm{~cm}$ Carl Zeiss Coudé solar telescope. The etalons were calibrated for the solar spectral lines Fe I $6173 \AA$, and Ca II $8542 \AA$. In this work, we discuss the characterization of the Fabry-Perot etalons, specifically the temperature and voltage tuning of the system for the spectral lines proposed for observations. We present the details of the calibration set-up and various tuning parameters. We also present solar images obtained using the system.
\end{abstract}

Keywords: Instrumentation and data management, Integrated Sun Observations, Spectral line

\section{Introduction}

Measuring the magnetic and velocity fields on the Sun is the key to understanding the solar activity and fine structure of sunspots, filaments, etc. One of the main techniques techniques for measuring solar magnetic and velocity fields involves spectroscopy, which can be carried out using either a high-resolution spectrograph or a tunable narrow-band filter in an imaging system (Zirin, 1995). Imaging spectroscopy allows us to obtain two-dimensional images (with certain field-of-view (FOV)) of the solar surface but requires scanning across multiple wavelength positions to build up the spectra. On the other hand, a slit-based

Udaipur Solar Observatory, Physical Research Laboratory,

Dewali, Badi road, Udaipur, India-313004

email: bayanna@prl.res.in 
spectrograph obtains the spectra but requires scanning across the FOV spatially to build up a two-dimensional image of the solar surface (Judge et al., 2010). In both cases, the observations from ground-based telescopes are affected by atmospheric turbulence. A number of post-facto techniques exist for imaging and imaging spectroscopy to deal with effects of atmospheric turbulence, but these techniques are still evolving for observations from a slit-based spectrograph (Beck, Rezaei and Fabbian, 2011).

Conventionally, narrow-band imaging and imaging spectroscopy can be carried out by using wavelength-tunable Lyot filters (Lyot, 1933: Evans, 1948: Stix, 1991) which can be slow because of mechanical movements involved in the tuning process. Using fast tunable narrow-band filters, two-dimensional images can be obtained by scanning across the spectral lines in shorter time periods (depending upon the number of wavelengths to be scanned along the line profile). Twodimensional images, with sparsely sampled spectral information, provide sufficient opportunity to use inversion techniques to retrieve the magnetic and velocity fields (Lagg et al., 2004, Borrero et al., 2011, Martínez Pillet et al., 2011). For example, the CRisp Imaging Spectropolarimeter (CRISP: Scharmer et al. (2008) ) installed at the Swedish 1-m telescope records Stokes images at 11 positions along the line profile of Fe I at $6302 \AA$ in steps of $48 \mathrm{~m} \AA$. The Stokes images were processed with the Milne-Eddington inversion software Helium Line Information Extractor (HELIX) (Lagg et al., 2004) to obtain the atmospheric parameters. SScharmer et al. (2008) used CRISP data to study the fine structure of the sunspot penumbra. Wiegelmann et al. (2010) also used the Stokes vector maps of the quiet Sun obtained with the Imaging Magnetograph eXperiment (IMaX) on the Sunrise (Barthol et al., 2011) telescope, to study the magnetic connectivity between the layers of the solar atmosphere. IMaX samples the line profile of $\mathrm{Fe} \mathrm{I}$ at $5250.2 \AA$ at a maximum of 12 positions. In this case,the inversion code Very Fast Inversion of the Stokes Vector (VFISV) developed by Borrero et al. (2011) is used to obtain atmospheric parameters. Wiegelmann et al. (2010) used the data to obtain the 3D structure of magnetic loops using extrapolation methods. Similarly, the Interferometric Bidimensional Spectrometer (IBIS) (Cavallini, 2006) installed at the Dunn Solar Telescope at Sacramento Peak (New Mexico, USA) records multiwavelength observations of the solar atmosphere to understand various solar phenomena. It scans the line profiles of $\mathrm{Fe}$ I, $\mathrm{H}_{\alpha}$, and $\mathrm{Ca}$ II at 14, 22, and 21 positions, respectively.

Some of the recent instruments use Fabry-Perot etalons to achieve narrowband imaging with a fast cadence (Burton, Leistner and Rust, 1987: Rust et al., 1996 Prasad Choudhary et al., 1998; Mathew et al., 1998: Kentischer et al., 1998: Tritschler et al., 2002: Cavallini, 2006: Gosain et al., 2006: Scharmer, 2006: Bello González and Kneer, 2008: Kleint, Feller and Gisler, 2011, Martínez Pillet et al., 2011). However, the electro-optically tunable solid-state etalons provide a smaller wavelength shift than air-spaced etalons for the same FOV because of their higher refractive index. This makes solid-state etalons of electro-optical materials such as lithium niobate (LN) useful for a larger FOV (Netterfield et al., 1997, Desai, 1996 Prasad Choudhary and Gosain, 2002 Martínez Pillet et al., 2011). After the successful use in ground-based and balloon-1 borne experiments, LN etalons have also been proposed for some of the future 
Table 1. Specifications of the Fabry-Perot etalons at $6328 \AA$ as provided by CSIRO, Australia.

\begin{tabular}{c|c|c|c|c|c|c|c|c}
\hline & $\mathrm{LiNb0}_{3}$ & $\begin{array}{c}\text { Clear } \\
\text { aperture }\end{array}$ & $\begin{array}{c}\mathrm{d}^{\perp} \\
(\mathrm{mm})\end{array}$ & $\begin{array}{c}\text { Refractive } \\
\text { index at } \\
6328 \AA\end{array}$ & $\begin{array}{c}\mathrm{R}^{\top} \\
(\%)\end{array}$ & $\begin{array}{c}\mathrm{FSR}^{\dagger} \\
(\AA)\end{array}$ & $\begin{array}{c}\mathrm{FWHM}^{\ddagger} \\
(\mathrm{m} \AA)\end{array}$ & Finesse \\
\hline $\mathrm{FP}_{1}$ & Z-cut & $60 \mathrm{~mm}$ & 0.226 & 2.286 & 93.30 & 3.6 & $\sim 150$ & $\sim 24.0$ \\
$\mathrm{FP}_{2}$ & Z-cut & $60 \mathrm{~mm}$ & 0.577 & 2.286 & 93.60 & 1.4 & $\sim 70$ & $\sim 20.0$ \\
\hline
\end{tabular}

Maximum recommended tuning voltage: $\pm 3000 \mathrm{~V}$

Maximum recommended rate of change of voltage: $\pm 1500 \mathrm{Vs}^{-1}$ Maximum recommended operating temperature: $45^{\circ} \mathrm{C}$

$\perp$ : Thickness, $\quad \top$ : Reflectivity, $\quad \dagger$ : Free spectral range, $\ddagger$ : Full-width at half maximum

space experiments such as Solar Orbiter (Mathew et al., 2001; Schuhle et al., 2007; Fahmy et al., 2013).

At the Udaipur Solar Observatory (USO), a narrow-band imaging system (imager) has been developed using two LN etalons in tandem with an appropriate thickness ratio. Using the etalons in tandem effectively increases the free spectral range (FSR) and the spectral resolution of the system (Kentischer et al., 1998; Tritschler et al., 2002). Although inter-reflections between multi-etalons cause ghost images, these can be eliminated or minimized either by tilting the etalons with respect to each other, or by placing a prefilter in between the etalons or by both (Tritschler et al., 2002, Kleint, Feller and Gisler, 2011; Martínez Pillet et al., 2011).

The etalons for the imager at USO were procured from the Common Wealth Scientific and Industrial Research Organization (CSIRO), Australia. Table 1 lists their relevant specifications. The imager will be used as a back-end instrument with the Multi-application Solar Telescope (MAST) (Denis et al., 2008, 2010. Mathew, 2009). MAST is an off-axis Gregorian a-focal telescope with a clear aperture of $50 \mathrm{~cm}$. This telescope was developed by Advanced Mechanical and Optical Systems (AMOS), Belgium, and has been installed at the lake site of USO, India. Along with a polarimeter, the imager will be used for measuring the magnetic and velocity fields of active regions in the photosphere and chromosphere. The initial aim is to carry out quasi-simultaneous observations in the two solar spectral lines Fe I at $6173 \AA$, and Ca II at $8542 \AA$, which are formed in the photosphere and chromosphere, respectively.

In this article, we discuss the calibration of the LN etalons in detail and evaluate their suitability for observations at the two spectral lines mentioned above. In Section 2 we discuss various tuning options for the LN etalons, and in Section 3 the optical set-up used to calibrate of these etalons is described. Results obtained from the voltage and temperature tuning of the system are explained in Section 4. Some of the preliminary images taken with the imager are shown in Section 5. 


\section{Lithium Niobate Fabry-Perot Etalon}

Fabry-Perot (FP) etalons produce a transmission spectrum (commonly known as channels) according to the Airy formula (Born and Wolf, 1989),

$$
I_{t}=\frac{1}{\left\{1+F \sin ^{2} \frac{\delta}{2}\right\}} I_{i}
$$

where $F=4 R /(1-R)^{2}, \delta=\frac{4 \pi}{\lambda} n_{o} d \cos \theta, R=\sqrt{r_{1} r_{2}}$. Here, $r_{1}$ and $r_{2}$ are the coefficients of the reflectance of the surfaces and $I_{i}$ and $I_{t}$ are the incident and transmitted intensities, respectively. $n_{o}, d$, and $\theta$ are the refractive index of the medium, spacing between the reflective surfaces, and the angle of refraction, respectively.

The working principle of the LN Fabry-Perot etalon is the same as that of any other etalon, except that the (partially) reflecting surfaces consist of highly polished faces of a LN wafer. Thus the crystal itself acts as a medium between the reflecting surfaces. To tune the etalon to different wavelengths, the electro-optic property of the LN substrate is used. Applying a voltage to this substrate in effect changes the refractive index of the crystal and thus produces a shift in the wavelength of the transmission channel (Yariv, 1984, Bonaccini and Smartt, 1988; Ghatak and Thyagarajan, 1989). The etalons used in this work are made of a birefringent, uni-axial z-cut LN crystal. The light propagating along the z-axis experiences a single refractive index $\left(n_{x}=n_{y}=n_{o}\right)$, which can be altered by the application of an electric field. The change in the refractive index $\left(\Delta n_{o}\right)$ (neglecting the effect of converse piezoelectric effect) is given by

$$
\Delta n_{o}=\frac{1}{2} n_{o}^{3} E_{z} r_{13}
$$

where $n_{o}$ is the refractive index of the ordinary ray at wavelength $\lambda_{0}, E_{z}$ is the electric field produced by the application of the voltage $(V)$, and $r_{13}$ is the relevant un-clamped electro-optic coefficient. The change in wavelength with the application of the voltage is given by,

$$
\delta \lambda=\frac{1}{2} \frac{n_{o}^{2} V r_{13} \lambda_{0}}{d}
$$

This is the most often used property of the LN crystal for tuning the filter to different wavelengths. The tuning rate is of the order of $200-500 \mathrm{~m}^{-1} \mathrm{~s}^{-1}$ depending on the thickness of the etalon. To tune the etalon for the entire wavelength range within two transmission peaks, the minimum voltage required is half the FSR voltage, which is given by $\lambda_{0} /\left(2 n_{o}{ }^{3} r_{13}\right)$. Half the FSR voltages estimated for $6173 \AA$ and $8542 \AA$ are around $5000 \mathrm{~V}$ and $8000 \mathrm{~V}$, respectively. This is more than the recommended safe voltage for LN etalons (see Table 1). The other two options for tuning the LN etalons are by varying the temperature and tilt of the etalons. Tilting the etalons changes the peak wavelength position, and for small angles the change in wavelength is given by $\delta \lambda=-\lambda \theta^{2} /\left(2 n_{o}{ }^{2}\right)$. 
In our experiment, the temperature tunability was used to fix the initial position of the transmission channel at the optimum wavelength position. Using voltage tuning, the desired spectral lines were then scanned. We have not explored the tilt tunability of the etalons in the present set-up. Sections 4.2 and 4.3 describe the temperature and voltage tuning of individual etalons, respectively, and Table 3 shows the values obtained after tuning. Section 4.4 describes the tuning of the etalons in tandem.

\section{Optical set-up for Calibration of Lithium Niobate Etalons and Narrow-band Imaging}

For the test and calibration of the LN etalons, we used a Littrow spectrograph set-up as shown in Figure 1, in conjunction with a Coudé telescope with a $15 \mathrm{~cm}$ clear aperture as the light feed. The F\#30 beam (see Figure 1) from the telescope was collimated and reimaged using lenses $\mathrm{L}_{1}$ and $\mathrm{L}_{2}$. Light passing through the slit (of width $100 \mu \mathrm{m}$ ) was collimated using a lens of focal length $150 \mathrm{~cm}$. The collimated light dispersed by a plane grating $(1200 \mathrm{l} / \mathrm{mm})$ was reimaged using the same Littrow lens. The spectrum obtained was magnified by a factor of two at the CCD plane using relay lenses L3 and L4. On the CCD, the dispersion per pixel (pixel size $=6.45 \mu \mathrm{m}$ ) is $17.45 \mathrm{~m} \AA$ and $14.85 \mathrm{~m} \AA$ at $6173 \AA$ and $8542 \AA$, respectively. The spectral coverage of the spectrograph is $17.8 \AA$ and $15.2 \AA$ at $6173 \AA$ and $8542 \AA$, respectively. The LN etalons $\left(\mathrm{FP}_{1}\right.$ and $\left.\mathrm{FP}_{2}\right)$ and the prefilter were introduced in the collimated beam after L1. For imaging, a beamsteering mirror (BSM) was inserted in the optical path before L2 to reflect the light toward a lens of focal length $75 \mathrm{~cm}$. This makes a final F\#30 beam, with an image scale of around 0.3 arcsec per pixel on the CCD camera.

Two different optical configurations are generally employed for imaging spectroscopy: a) FP etalons in a collimated beam near the re-imaged pupil plane, and b) FP etalons in a telecentric configuration. The merits and demerits of both configurations have been explained by Cavallini (2006) and Scharmer (2006).

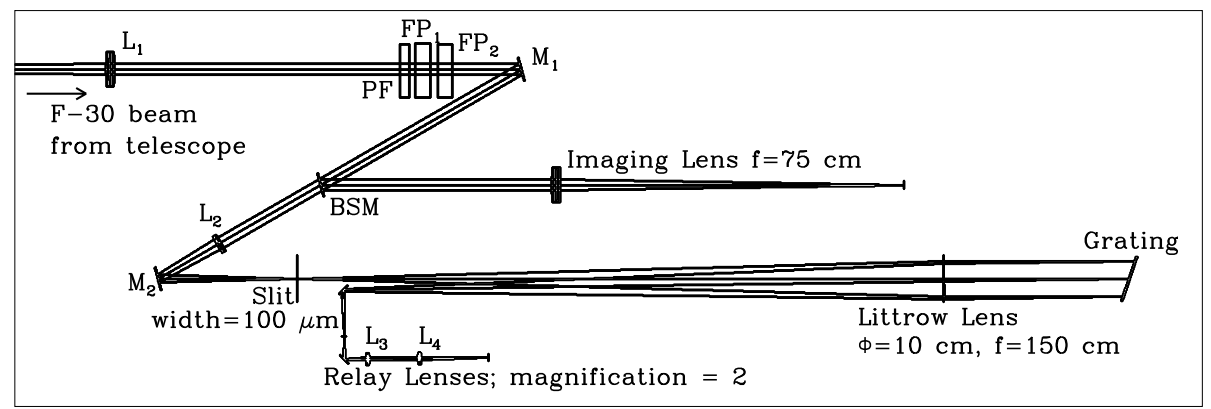

Figure 1. Optical layout of the spectrograph and imaging set-up. BSM is the beam-steering mirror for directing the light transmitted from the narrow-band filter $\left(\mathrm{FP}_{1}\right.$ and $\left.\mathrm{FP}_{2}\right)$ toward the imaging set-up. The prefilter (PF) wheel consists of two prefilters centered on $6173 \AA$ and $8542 \AA . M_{1}$ and $M_{2}$ are folding mirrors. 

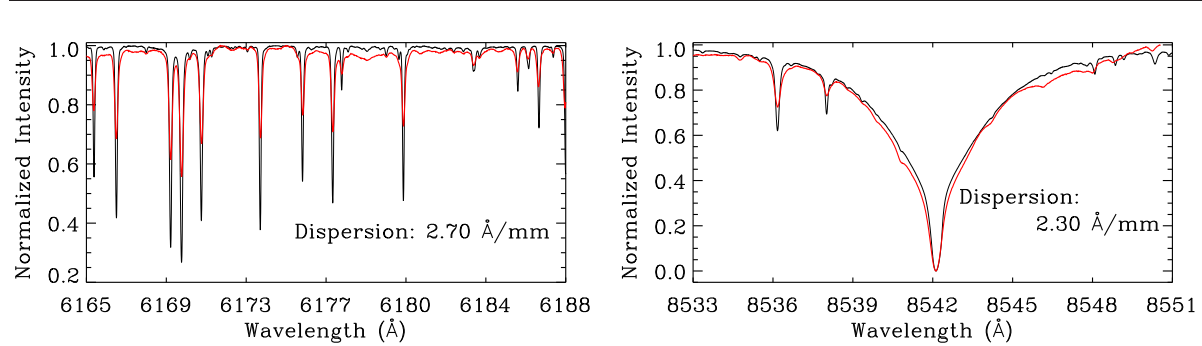

Figure 2. Left and right panels show a comparison of the spectrum (red continuous line) obtained using the optical set-up described in Figure 1 with that obtained from the BASS2000 archive (black continuous line) for the $6173 \AA$ and $8542 \AA$ lines, respectively.

However, as our primary goal is to characterize the etalons, we preferred a collimated set-up 1 . A maximum blue shift of $11 \mathrm{~m} \AA$ is expected at $6173 \AA$ due to the collimated set-up. Figure 2 shows the plots of the solar spectrum obtained with our spectrograph set-up and that taken from the BASS20002 solar survey spectral atlas for comparison.

\section{Characterization of the LN Etalons}

Channel spectra of both LN etalons were recorded by placing them in a collimated beam after lens L1. Figure 3 shows the spectra obtained with the optical set-up. The top panel show the solar spectra, which include the $6173 \AA$ (left) and $8542 \AA$ (right) spectral lines. The remaining three panels, from top to bottom, show the transmission channels for $\mathrm{FP}_{1}$ (thinner, $226 \mu \mathrm{m}$ ), $\mathrm{FP}_{2}$ (thicker, 577 $\mu \mathrm{m})$ and the FPs in tandem in logarithmic scale. It is worth noting that the FSR (wavelength separation between two transmission peaks) increases for the tandem configuration. From these measurements, we computed various parameters for the FPs that are essential for using them in the imager set-up at specific

${ }^{1}$ Telecentric configuration with $\mathrm{F} \# \approx 100$ will be chosen in the future for solar observations using this instrument with MAST.

${ }^{2}$ BASS2000: Solar survey archive, http : //bass2000.obspm. fr , Delbouille, Roland and Neven 1973

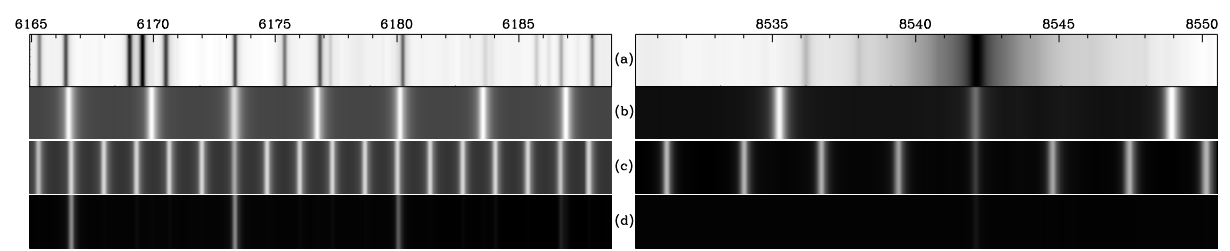

Figure 3. (a) Observed solar spectrum, (b) channel spectrum of $\mathrm{FP}_{1}$, (c) channel spectrum of $\mathrm{FP}_{2}$, (d) channel spectrum of the FPs in tandem near the crossover voltage (the voltage where the channel crosses the line center). Left panels correspond to the 6173 A line, right panels to the $8542 \AA$ lines. The intensity in panels (b), (c), and (d) are in logarithmic scale. 
Table 2. Measured values of the FSR and FWHM of the FPs

\begin{tabular}{|c|c|c|c|c|}
\hline & Parameter & FP1 & FP2 & Tandem \\
\hline \multirow[t]{3}{*}{$6173 \AA$} & & & & \\
\hline & FSR $(\AA)$ & 3.400 & 1.350 & 6.700 \\
\hline & FWHM (mA) & 196.8 & 154.0 & 150.3 \\
\hline \multirow[t]{3}{*}{$8542 \AA$} & & & & \\
\hline & $\operatorname{FSR}(\AA)$ & 6.830 & 2.690 & ¿10.0 \\
\hline & FWHM (mA) & 273.3 & 190.0 & 190.6 \\
\hline
\end{tabular}

wavelengths. Some of the important estimated parameters are the FSR, the full width at half maximum (FWHM), and voltage and temperature tuning rates. In the following sections, we discuss the methodology adopted to obtain these parameters.

\subsection{Free Spectral Range and Full Width at Half Maximum}

The recorded channel spectra were analyzed to obtain the FSR and the FWHM for the FPs individually and in tandem. The central 200 pixels of the recorded spectrum corresponding to a FOV of one arcmin were averaged in the slit direction to obtain the transmission profile of the etalons. The wavelength scale was calculated from the solar spectra, which were recorded before placing the etalons in the beam path. We have used almost all the channels in our recorded channel spectrum to compute the mean FSR and FWHM. Table 2 shows the measured values of these parameters. Although the FSR obtained nearly matches the values specified by the vendor, we observed a larger FWHM than for the specified values. The difference is mainly attributed to the low spectral resolution due to the larger width of the spectrograph slit. We estimated the FWHM of the spectrograph $\left(\Delta \lambda_{s}\right)$ from the FWHMs of the recorded $6173 \AA$ line profile $\left(\Delta \lambda_{r}\right)$ and that obtained from the BASS2000 atlas $\left(\Delta \lambda_{c}\right)$ using the relation $\left(\Delta \lambda_{r}\right)^{2}=\left(\Delta \lambda_{s}\right)^{2}+\left(\Delta \lambda_{c}\right)^{2}$. The FWHM of the spectrograph is estimated to be about $140 \mathrm{~m} \AA$. Thus, the measured FWHMs are (roughly) consistent with the specifications in Table 1, and with the actual resolution of the spectrograph.

\subsection{Temperature tuning of the LN etalons}

The refractive index of the $\mathrm{LN}$ is sensitive to temperature. To avoid any wavelength shift during observations, we placed the etalons in temperature-stabilized enclosures. These enclosures keep the etalons within $\pm 0.05^{\circ} \mathrm{C}$ around the set temperature to maintain a wavelength stability of about $\pm 1.2 \mathrm{~m} \AA$.

The temperature of the enclosure can be set to between $23^{\circ} \mathrm{C}$ and $45^{\circ} \mathrm{C}$. Temperature-sensitivity measurements were carried out by setting the temperature of the enclosure between $38^{\circ} \mathrm{C}$ to $23^{\circ} \mathrm{C}$, in steps of $-3^{\circ} \mathrm{C} .30$-minute interval between each step was allowed for the temperature to stabilize to the set value. At each temperature, voltage was applied to the FPs from $-3 \mathrm{kV}$ to $+3 \mathrm{kV}$ in 

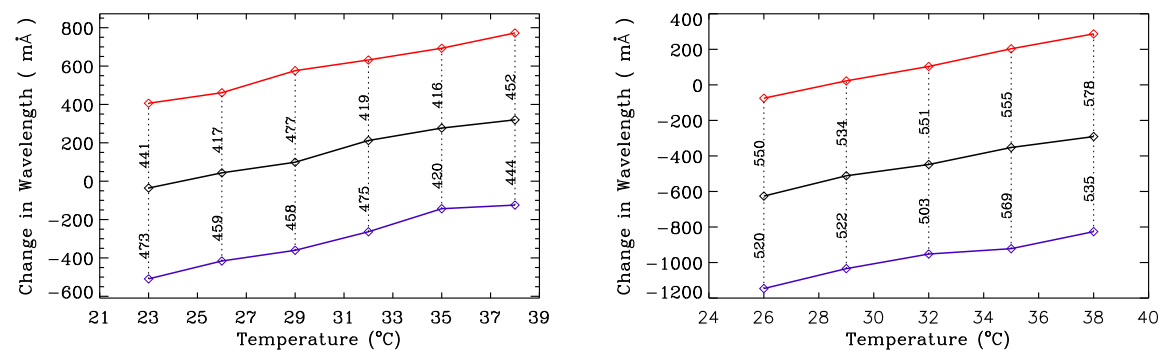

Figure 4. Left and right panels show the temperature-sensitivity of $\mathrm{FP}_{2}$ at $6173 \AA$ and 8542 A, respectively. Blue, black, and red continuous lines joining the diamonds represent the shift in wavelength with temperature for $-3 \mathrm{kV}, 0 \mathrm{~V}$, and $+3 \mathrm{kV}$, respectively. The values between the curves indicate the change in wavelength (in $\mathrm{m} \AA$ ) corresponding to the change in voltage at a given temperature. The average slope is around $25 \mathrm{~m}^{\circ} \mathrm{C}^{-1}$.

steps of $100 \mathrm{~V}$ and the corresponding shift in the wavelength of the transmission channel was measured. We also estimated the crossover voltage $(\mathrm{CoV})$, i.e. the voltage where the transmission channel crosses the center of the solar spectral line.

Figure 4 shows the results of these measurements for etalon $\mathrm{FP}_{2}$. We plotted the shift in wavelength of the transmission in function of temeperature, close to the spectral lines of interest centered on $6173 \AA$ (left) and $8542 \AA$ (right). Blue, black, and red continuous lines joining the diamonds represent the shift in wavelength from line center for $-3 \mathrm{kV}, 0 \mathrm{~V}$, and $+3 \mathrm{kV}$, respectively. An increase in the temperature moves the transmission peaks to the higher wavelength side, indicating an increase in the refractive index of the LN etalon.

From Figure 4 it is clear that the optimum temperature of $\mathrm{FP}_{2}$ is $35^{\circ} \mathrm{C}$. At this temperature, the transmission channel of $\mathrm{FP}_{2}$ can scan both line profiles optimally with the application of the voltage in the range of $\pm 3 \mathrm{kV}$. Similarly, the temperature tuning of $\mathrm{FP}_{1}$ shows that the etalon kept at any temperature between $27^{\circ} \mathrm{C}$ and $33^{\circ} \mathrm{C}$ would allow us to scan the full line profile of both lines by applying voltages in the range of $\pm 3 \mathrm{kV}$.

\subsection{Voltage Tuning of the LN Etalons}

In this section, we explore how the voltage tunability of the etalons can be used to scan the two line profiles while keeping the etalons at an optimum temperature. As mentioned earlier, voltage tuning of the etalons is widely used to obtain high-cadence, multiwavelength observations. A linear high-voltage power supply with zero-crossing (from Applied Kilovolts, UK, now a part of Exelis Inc., USA) was used to obtain an output of $\pm 5 \mathrm{kV}$ for a computer-controlled input of $\pm 10 \mathrm{~V}$ in direct current (DC). The details of the power supply unit and the control electronics can be found in Mathew and Gupta (2010). The etalons are operated by restricting the voltage between $\pm 3 \mathrm{kV}$ and the rate of change of the voltage across the etalons is maintained at less than $1.5 \mathrm{kVs}^{-1}$. These values are 

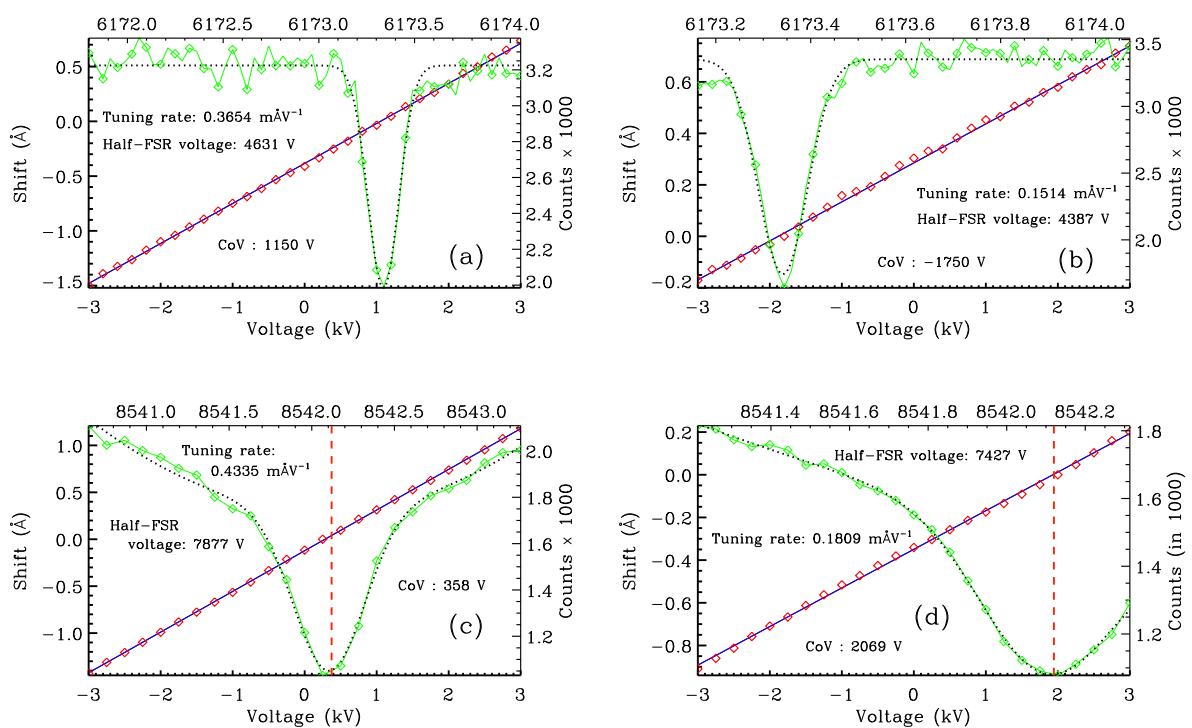

Figure 5. Voltage tuning of the individual etalons at the lines of interest: (a) and (b) for $\mathrm{FP}_{1}$ and $\mathrm{FP}_{2}$ at $6173 \AA$, respectively; (c) and (d) for $8542 \AA$ line, respectively. The panels show (i) the wavelength shift from the line center as a function of voltage with a blue continuous line and red diamonds and (ii) scanning of the spectral line with the application of the voltage to the individual etalons, which is shown as a green continous line with diamonds corresponding to the data counts vs. voltage. A Gaussian fit of this curve is shown as a black dotted line. The red vertical dashed line represents the line center. The corresponding wavelength axis is shown at the top of each panel. It is evident that for $6173 \AA, \mathrm{FP}_{2}$ has to be maintained at a temperature lower than $35^{\circ} \mathrm{C}$, whereas for $8542 \AA$, a higher temperature $\left(\geq 35^{\circ} \mathrm{C}\right)$ is required.

restricted according to the CSIRO advice for safe operation of the etalons and are taken into account in the control software.

Figures $5 \mathrm{a}-\mathrm{d}$ show the results obtained from the voltage tuning of both etalons for the two spectral lines by keeping the etalons $\mathrm{FP}_{1}$ and $\mathrm{FP}_{2}$ at a temperature of $30^{\circ} \mathrm{C}$ and $35^{\circ} \mathrm{C}$, respectively. The measurements were carried out by recording the transmission channel spectra for voltages from $+3 \mathrm{kV}$ to $-3 \mathrm{kV}$ with a step size of $100 \mathrm{~V}$. The red diamonds represent the measured wavelength shift from $\lambda_{0}$ for the applied voltages (x-axis). The straight continuous line fitting the measurements allows us to estimate the voltage-tuning rate and the half-FSR voltage. The crossover voltage $(\mathrm{CoV})$, i.e. the voltage at which the transmission channel crosses the center of the spectral line, is obtained from the green curve shown in Figure 5.

The voltage-tuning rate obtained from these measurements is indicated in the plots in units of $\mathrm{m} \AA \mathrm{V}^{-1}$ along with the required half-FSR voltages. The estimated tuning rate $(\delta \lambda / V)$ allows us to compute the unclamped electro-optic coefficient $\left(r_{13}\right)$ of the LN crystals using Equation (3). The mean value of $r_{13}$ for $\mathrm{FP}_{1}$ and $\mathrm{FP}_{2}$ at $6173 \AA$ is estimated as $5.08 \pm 0.06 \mathrm{pmV}^{-1}$ and $5.30 \pm 0.15 \mathrm{pmV}^{-1}$ respectively, while the mean value of the $r_{13}$ coefficient at $8542 \AA$ is estimated 
A. Raja Bayanna et al.

Table 3. Parameters obtained by temperature and voltage tuning of the etalons

\begin{tabular}{c|c|c|c|c|c|c|c}
\hline $\begin{array}{c}\text { Wave- } \\
\text { length }\end{array}$ & $\begin{array}{c}\text { Fabry- } \\
\text { Perot }\end{array}$ & $\begin{array}{c}\text { Optimum } \\
\text { Temperature }\end{array}$ & $\begin{array}{c}\mathrm{VT}^{\perp} \\
\left(\mathrm{mA} \mathrm{V}^{-1}\right)\end{array}$ & $\begin{array}{c}\mathrm{CoV}^{\top} \\
(\mathrm{V})\end{array}$ & $\begin{array}{c}\mathrm{V}_{\frac{1}{2} F S R} \\
(\mathrm{~V})\end{array}$ & $\begin{array}{c}\text { Tuning } \\
\text { ratio }\end{array}$ & $\begin{array}{c}r_{13} \\
\left(\mathrm{pmV}^{-1}\right)\end{array}$ \\
\hline $6173 \AA$ & $\mathrm{FP}_{1}$ & $30^{\circ} \mathrm{C}$ & 0.36541 & +1150 & 4631 & 2.413 & 5.08 \\
& $\mathrm{FP}_{2}$ & $35^{\circ} \mathrm{C}$ & 0.15147 & -1750 & 4387 & & 5.30 \\
\hline $8542 \AA$ & $\mathrm{FP}_{1}$ & $30^{\circ} \mathrm{C}$ & 0.43353 & +0358 & 7877 & 2.396 & 4.37 \\
& $\mathrm{FP}_{2}$ & $35^{\circ} \mathrm{C}$ & 0.18094 & +2069 & 7427 & & 4.64 \\
\hline$\perp:$ Voltage tuning rate, $\mathrm{T}:$ Crossover Voltage, $V_{\frac{1}{2} F S R}:$ Half-FSR voltage
\end{tabular}

to be $4.37 \mathrm{pmV}^{-1}$ and $4.64 \pm 0.06 \mathrm{pmV}^{-1}$ for $\mathrm{FP}_{1}$ and $\mathrm{FP}_{2}$, respectively. The $r_{13}$ coefficients estimated from voltage-tuning rates obtained at different temperatures were averaged to obtain the mean value of the coefficient. Different parameters obtained from temperature and voltage tuning of the etalons are shown in Table 3. The observed difference (244 V at $6173 \AA$ and $450 \mathrm{~V}$ at $8542 \AA$ ) in the half-FSR voltages of $\mathrm{FP}_{1}$ and $\mathrm{FP}_{2}$ at a given wavelength might be due to the variation in the $r_{13}$ coefficients of $\mathrm{FP}_{1}$ and $\mathrm{FP}_{2}$ introduced during the crystal growth process.

In this regard, we note that the values of the $r_{13}$ coefficients reported by various authors differ considerably. Mathew (2003) estimated the $r_{13}$ coefficient of a $z$-cut LN crystal to be $6.28 \mathrm{pmV}^{-1}$ at $6122 \AA$. This value differs from those obtained by other researchers. For example, Turner (1966) and Onuki, Uchida and Saku (1972) reported a value of $8.6 \mathrm{pmV}^{-1}$ and $10.9 \mathrm{pmV}^{-1}$ measured at $6328 \AA$, respectively. Similarly, Burton, Leistner and Rust (1987) reported a value of $6.14 \mathrm{pmV}^{-1}$ at $5800 \AA$. Most of these values correspond to different wavelengths and, hence, it would be interesting to study the wavelength dependence of $r_{13}$.

As evident from Figure 5, the half-FSR voltages required to tune the etalons anywhere between the FSR are higher than the safe voltage that can be applied to the crystal. This makes it difficult to tune the etalons by applying a voltage to scan the entire line profile of Ca II $8542 \AA$. It should be possible to specify the central wavelength position of one of the channels to be exactly in the line center when fabricating the etalons. However, using the same etalon for multiwavelength observations is very difficult. Furthermore, for a broad spectral line like Ca II $8542 \AA$, the restriction in the applied voltage allows us to scan only a part of the line profile. The other possibility of tuning the etalons by varying their temperatures is also constrained by the recommended maximum operating temperature of $45^{\circ} \mathrm{C}$. We have not explored the tilt tuning of the etalons. Moreover, as the reflective coatings of the etalons are optimized for normal incidence, reflection losses are expected at larger tilt and, thus, the effective finesse of the etalons reduces. Therefore, tilt tuning is not a good option.

\subsection{Voltage Tuning of the LN Etalons in Tandem}

The etalons were tuned in tandem using the values obtained after temperature and voltage tuning of the individual etalons. The temperature for $\mathrm{FP}_{1}$ and $\mathrm{FP}_{2}$ 
was fixed at $30^{\circ} \mathrm{C}$ and $35^{\circ} \mathrm{C}$, respectively. The voltage-tuning values indicated in Figure 5 and listed in Table 3 were used to tune the etalons in tandem to scan the line profiles. Crossover voltages of the individual etalons were used to obtain the transmission profile of the FPs in tandem at the center of the lines of interest, and the voltage-tuning rates were used to set the transmission peaks of both etalons to the same wavelength. For example, the ratio of the voltage-tuning rates of $\mathrm{FP}_{1}$ and $\mathrm{FP}_{2}$ at $6173 \AA$ and $8542 \AA$ are 2.413 and 2.396, respectively. This means that $\mathrm{FP}_{2}$ requires $2.413 \mathrm{~V}$ for each $1 \mathrm{~V}$ change of $\mathrm{FP}_{1}$ to shift the transmission channel to the same wavelength position. Similarly, it requires $2.396 \mathrm{~V}$ for observations at $8542 \AA$. The scanning of the spectral line in any desired wavelength step can be carried out by a control program provided the voltage tuning rates and crossover voltages are known.

\section{Preliminary Observations with the Narrow-Band Imager}

After carrying out the calibration runs using the spectrograph, the collimated beam from the FPs was diverted toward the imaging system using a beamsteering mirror (BSM) as described in Section 3. A filter wheel with two interference filters centered on $6173 \AA$ (FWHM $=3.0 \AA$ ) and $8542 \AA$ (FWHM = $3.5 \AA$ ) was employed to select the desired wavelength (channel) for observations. A series of images was obtained by tuning the etalons in tandem across different wavelength positions on both spectral lines. Images acquired at different wavelength positions are shown in Figure 6. Figure 7 shows a magnified view of the images taken in the line center at $6173 \AA$ and $8542 \AA$, respectively. Observations in $6173 \AA$ were obtained on 3 April 2013, the sunspot observed was part of the active region (AR) NOAA 11711 located at S17E24 on the solar disk. Observations in $8542 \AA$ were obtained on 8 March 2013, the filament that is seen in the image was located on the disk at S35E25 near AR 11689.

For the $6173 \AA$ line, the images were obtained from $-150 \mathrm{~m} \AA$ to $+210 \mathrm{~m} \AA$ from line center, with a step of $30 \mathrm{~m} \AA$, whereas the line profile at $8542 \AA$ was scanned from $-225 \mathrm{~m} \AA$ to $+150 \mathrm{~m} \AA$ in a step of $75 \mathrm{~m} \AA$. It is noteworthy here
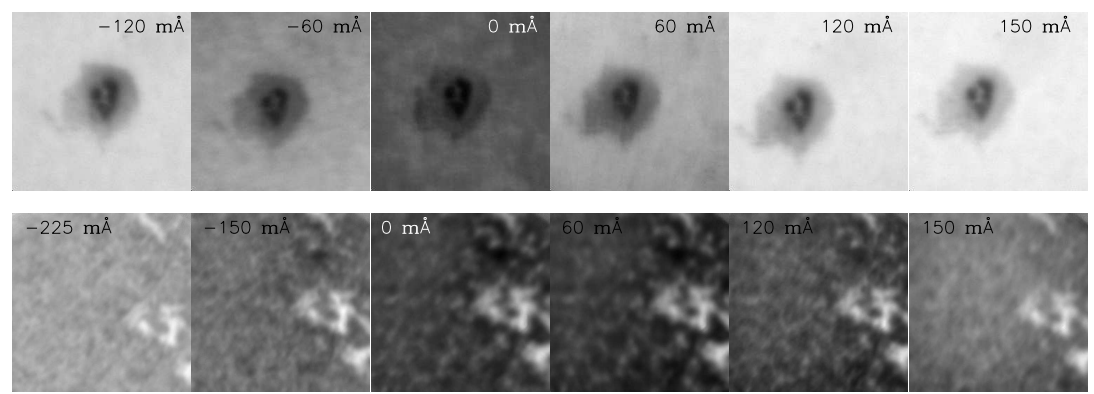

Figure 6. Images obtained at $6173 \AA$ (top) and $8542 \AA$ (bottom), at the indicated wavelength positions during the corresponding line scan. The FOV for the top panels is $150 \operatorname{arcsec}^{2}$ and that for the bottom panels is $300 \operatorname{arcsec}^{2}$. 

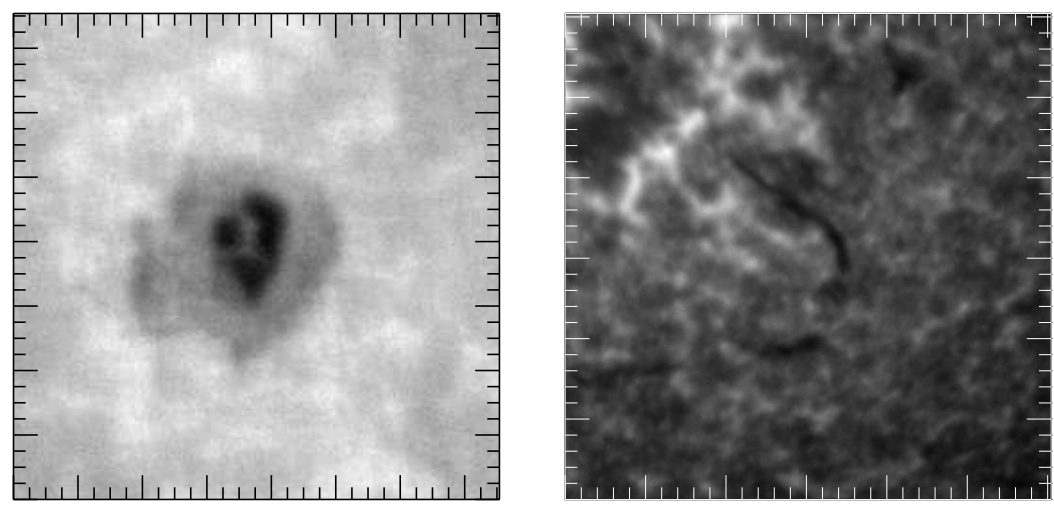

Figure 7. Images obtained by tuning the filter on the line center of (a) $6173 \AA$ and (b) $8542 \AA$. The FOV of (a) is $150 \operatorname{arcsec}^{2}$ and of (b) $300 \operatorname{arcsec}^{2}$.

that the quality of these images is affected by the poor seeing conditions at the sit 3 . Using similar observations obtained for the quiet Sun, we retrieved the profiles for both spectral lines (Figure 8). The mean intensity of the quiet Sun after flat-fielding and dark-current correction were used in retrieving these line profiles. As evident, because of the limited tunability of the filter, we were unable to retrieve the complete line profile of the $8542 \AA$ line. The exposure times used to obtain each filtergram in $6173 \AA$ and $8542 \AA$ are $120 \mathrm{~ms}$ and $900 \mathrm{~ms}$, respectively. However, with a $50 \mathrm{~cm}$ telescope the exposure times are expected to be of the order of $50 \mathrm{~ms}$ and $300 \mathrm{~ms}$, respectively, to build the signal to a level of $80 \%$ of the full-well capacity (i.e. $16000 \mathrm{e}^{-}$) of the CCD.
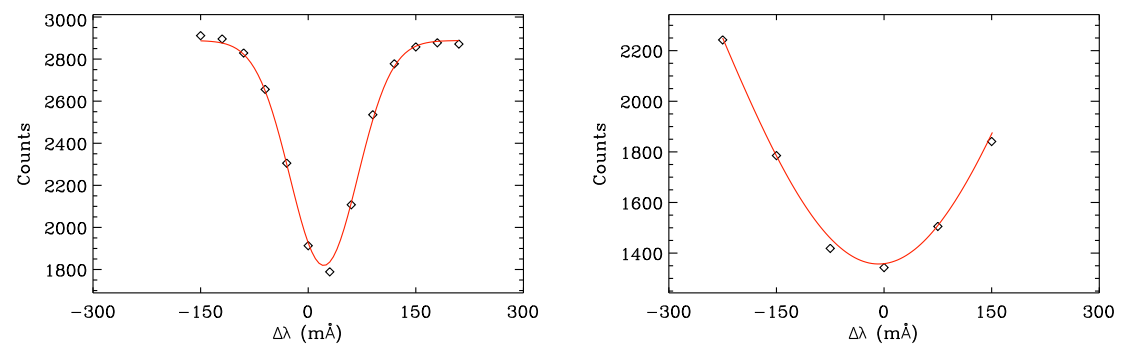

Figure 8. Retrieved line profiles at $6173 \AA$ (left) and $8542 \AA$ (right) using quiet-Sun observations. The line profile at $6173 \AA$ is scanned at 13 wavelength positions from $-150 \mathrm{~m} \AA$ to +210 $\mathrm{m} \AA$ with $\delta \lambda=30 \mathrm{~m} \AA$. The line profile at $8542 \AA$ is scanned only in the core at five wavelength positions from $-225 \mathrm{~m} \AA$ to $+150 \mathrm{~m} \AA$ with $\delta \lambda=75 \mathrm{~m} \AA$.

${ }^{3}$ The $15 \mathrm{~cm}$ Coudé telescope used for calibration and acquiring test images is located next to a laboratory building. However, the narrow-band imager will be integrated with a $50 \mathrm{~cm}$ telescope MAST at the island observatory, where the seeing conditions are relatively better. 


\section{Conclusions}

We demonstrated the use of two lithium niobate etalons $\left(\mathrm{FP}_{1}\right.$ and $\left.\mathrm{FP}_{2}\right)$ in tandem for quasi-simultaneous narrow-band observations in Fe I (6173 $\mathrm{A})$ and Ca II $(8542 \AA)$. Temperature and voltage-tuning calibrations for both etalons were carried out using a Littrow spectrograph. The temperatures of $\mathrm{FP}_{1}$ and $\mathrm{FP}_{2}$ were fixed at $30^{\circ} \mathrm{C}$ and $35^{\circ} \mathrm{C}$, respectively. The ratio of voltage-tuning rates of $\mathrm{FP}_{1}$ and $\mathrm{FP}_{2}$ at $6173 \AA$ and $8542 \AA$ were found to be 2.413 and 2.396, respectively. The computed values of the $r_{13}$ coefficients for the lithium niobate crystals were found to be of the order of $5.2 \mathrm{pmV}^{-1}$ and $4.5 \mathrm{pmV}^{-1}$ at $6173 \AA$ and $8542 \AA$, respectively.

A Coudé telescope with a clear aperture of $15 \mathrm{~cm}$ was used to obtain the test images in both lines. The line profiles of these two lines of interest were retrieved using quiet-Sun observations. However, given the limitations, the system can scan the $8542 \AA$ line only partially. This system will be integrated with the upcoming solar telescope, MAST, in about mid-2014. We did not perform a rigorous ghost analysis here. We plan to use a prefilter in between the two etalons to deal with the ghost images after integrating the instrument with MAST. The ghost-image analysis will be carried out for the system, and if required, the etalons will be tilted to minimize the problem by avoiding any significant broadening of the filter profile (Martínez Pillet et al., 2011).

Acknowledgements We acknowledge the work done by S. K. Gupta to develop the control electronics for the high-voltage power-supply unit and temperature controller. We also acknowledge the work done by M. Sardava for the design and fabrication of the temperaturecontrolled enclosures for the FPs and the prefilter wheel, is also acknowledged. We thank the anonymous referee for his/her valuable suggestions and comments that improved the quality of the article.

\section{References}

Barthol, P., Gandorfer, A., Solanki, S.K., Schüssler, M., Chares, B., Curdt, W., et al..: 2011, Solar Phys. 268, 1.

Beck, C., Rezaei, R., Fabbian, D.: 2011, Astron. Astroph. 535, A129.

Bello González, N. and Kneer, F.: 2008, Astron. Astroph. 480, 265.

Bonaccini, D., Smartt, R., N.: 1988, Appl. Opt. 27, 5095.

Born, M., Wolf, E.: 1989, Principles of Optics, Pergamon Press.

Borrero, J.M., Tomczyk, S., Kubo, M., Socas-Navarro, H., Schou, J., Couvidat, S., Bogart, R.: 2011, Solar Phys. 273, 267.

Burton, C. H., Leistner, A. J., Rust, D. M.: 1987, Appl. Opt. 26, 2637.

Cavallini, F.: 2006, Solar Phys. 236, 415.

Desai, J. N.: 1996, Bull. Astron. Soc. India 24, 849.

Delbouille, L., Roland, G., Neven, L.: 1973, Atlas photometrique du spectre solaire de $\lambda 3000$ a $\lambda 10$ 000, Universite de Liege, Institut d' Astrophysique, Liege.

Denis, S., Coucke, P., Gabriel, E. et al.: 2008, Proc. SPIE 7012, id. 701235.

Denis, S., Coucke, P., Gabriel, E. et al.: 2010, Proc. SPIE 7733, id. 773335.

Evans, J. W.: 1948, J. Opt. Soc. Am. 30, 229.

Fahmy, S. et al.: 2013 Proc. 64 Int. Astron. Con. IAC-13, A3.5.2

Gosain, S., Venkatakrishnan, P., Venugopalan, K.: 2006, J. Astrophys. Astron. 27, 285.

Ghatak, A., Thyagarajan, K.: 1989, Optical electronics, Cambridge University Press. 
Judge, P.G., Tritschler, A., Uitenbroek, H., Reardon, K., Cauzzi, G., de Wijn, A.: 2010, Astrophys. J. 710, 1486.

Kentischer, T.J., Schmidt, W., Sigwarth, M., and Uexkuell, M.V.: 1998, Astron. Astroph. 340, 569.

Kleint, L., Feller, A., Gisler, D.: 2011, Astron. Astroph. 529, A78.

Lagg, A., Woch, J., Krupp, N., Solanki, S. K.: 2004, Astron. Astroph. 414, 1109.

Lyot, B.: 1993, Compt Rend. Acad. Sci. Paris 197, 1593.

Martínez Pillet, V., Del Toro Iniesta, J.C., Álvarez-Herrero, A., Domingo, V., Bonet, J.A., González Fernández, et al.: 2011, Solar Phys. 268, 57.

Mathew, S. K., Bhatnagar, A., Prasad, C. D., Ambastha, A.: 1998, Astron. Astrophys. Suppl. 133, 285.

Mathew, S. K., Solanki, S. K., and VIM team.: 2001, in S. C. Tripathy, P. Venkatakrishnan (ed.) Probing the Sun with high resolution, Narosa Pub. House, 213.

Mathew, S. K.: 2003, Appl. Opt. 42, 3580.

Mathew, S. K.: 2009, in S.V. Berdyugina, S.K., Nagendra, R. Ramelli (ed.) Solar Polarization 5: In Honor of Jan Stenflo 405, 461.

Mathew, S. K., Gupta, S. K.: 2010, Tech. Rep. PRL-TN-2010-97, Phys. Res. Lab., Ahmedabad, India.

Netterfield, R. P., Freund, C. H, Seckold J. A., Walsh, C. J.: 1997, Appl. Opt. 36, 4556.

Onuki, K., Uchida, N., Saku, T.: 1972, J. Opt. Soc. Am. 62, 1030.

Prasad Choudhary, D., Gosain, S.: 2002, Exp. Astron. 13, 153.

Prasad Choudhary, D., Mathew, S., Bhatnagar, A., Ambastha, A.: 1998, Exp. Astron. 8, 125.

Rust, D.M., Murphy, G., Strohbehn, K., Keller, C.U.: 1996, Solar Phys. 164, 403.

Scharmer, G. B.: 2006, Astron. Astroph. 447, 1111.

Scharmer, G. B., Narayan, G., Hillberg, T., de la Cruz Rodríguez, J., Löfdahl, M.G., Kiselman, D., et all.: 2008, Astrophys. J. 689, L69.

Schühle, U., Mathew, S.K., Wedemeier, M., Hartwig, H., Ballesteros, E., Martinez Pillet, V., Solanki, S.K.: 2007, ESA Special Publication 641, 82.

Stix, M.: 1991, The Sun: An Introduction, Springer-Verlag.

Tritschler, A., Schmidt, W.: Langhans, K., Kentischer, T.: 2002, Solar Phys., 211, 17.

Turner, E. H.: 1966, Appl. Phys. Lett. 8, 303.

Wiegelmann, T., Solanki, S.K., Borrero, J.M., Martínez Pillet, V., del Toro Iniesta, J.C., Domingo, V., et al.: 2010, Astrophys. J. 723, L185.

Yariv, A.: 1984, Optical electronics, Holt McDougal, Third Ed.

Zirin, H.: 1995, Solar Phys. 159, 203. 